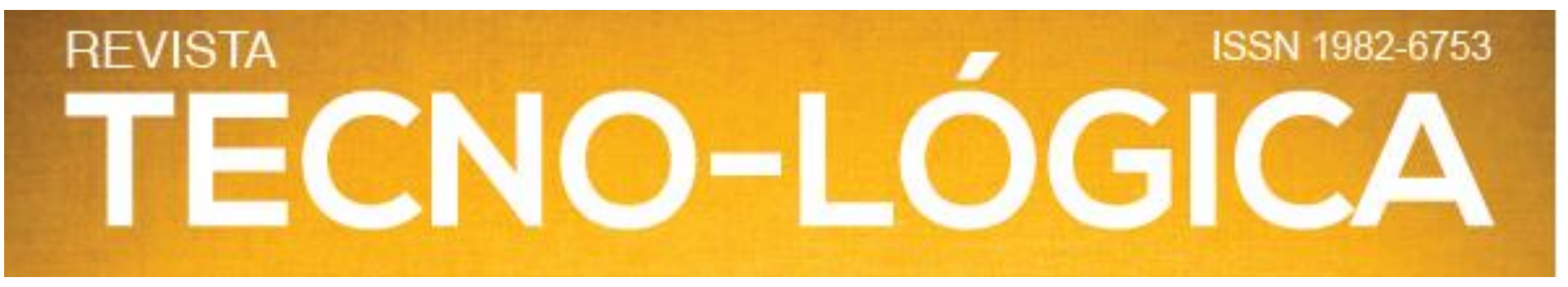

\title{
REVISÃO SOBRE O LIXO ELETRÔNICO E SEU DESTINO NA CIDADE DE SANTA CRUZ DO SUL, RS, BRASIL
}

\author{
Luiz Henrique Rauber Rodrigues ${ }^{1 *}$, Victor Hugo Salinas Camarillo², Eduardo Alexis Lobo ${ }^{1}$, Enio Leandro Machado ${ }^{1-3}$ \\ ${ }^{1}$ Programa de Pós-graduação em Tecnologia Ambiental, Universidade de Santa Cruz do Sul (PPGTA/UNISC), RS, Brasil. \\ ${ }^{2}$ Doutorado em Ciencias del Mar y Limnología, Universidad Nacional Autónoma de México, México. \\ ${ }^{3}$ Programa de Pós-graduação em Sistemas e Processos Industriais, Universidade de Santa Cruz do Sul (PPGSPI/UNISC), RS, Brasil. \\ *E-mail: luizhrr@gmail.com \\ Recebido em: 05/07/2020 \\ Aceito em: 22/04/2021 \\ DOI:10.17058/tecnolog.v25i2.15407
}

\section{RESUMO}

Os produtos eletrônicos descartados incorretamente podem causar muitos danos ambientais e, consequentemente, à saúde humana. Nesse contexto, foi realizada uma revisão teórica das leis e normas brasileiras sobre resíduos sólidos eletrônicos, empresas que atuam na área e a avaliação do descarte de resíduos eletrônicos na cidade de Santa Cruz do Sul, RS, além da potencial revalorização de computadores descartados localmente. Verificamos que existem soluções para a disposição de resíduos eletrônicos de pessoas físicas e jurídicas, principalmente empresas de informática da cidade, destacando que para as primeiras existe um local adequado na cidade denominado CEPREL (Centro de Coleta de Pneus e Lixo Eletrônico), mantido pela Prefeitura Municipal. As empresas têm seus próprios processos. Consideramos como benefício social que uma das melhores soluções municipais é o BTEC (Banco Social de Tecnologia do Vale do Rio Pardo), que coleta computadores desativados de empresas, os repara e doa a escolas e entidades beneficentes.

Palavras-chave: Tecnologia Ambiental; Lixo Eletrônico; Descarte; Revalorização; TI Verde.

\section{Introdução}

A área de Tecnologia da Informação e Comunicação (TIC) assim como todo o ecossistema envolto, normalmente denominado como "informática", traz muitos benefícios de conhecimento público. Exemplos são a facilidade de comunicação, a melhora na velocidade e automatização de tarefas, contribuições para a realização e divulgação de processos e resultados, tanto profissionais como acadêmicos.

Dentro deste ambiente da informática e seus inúmeros jargões existentes, um que tem tido destaque é a da TI Verde ou Green IT. Este é um movimento iniciado para propor mais sustentabilidade às áreas envolvidas com tecnologia, como a redução de desperdício, aumento da eficiência no uso de computadores, reutilização de recursos tecnológicos, a conscientização socioambiental e o descarte adequado do resíduo eletrônico ("lixo eletrônico", ou e-lixo ou e-waste) [1-7]. O lixo eletrônico são todos os resíduos resultantes da rápida obsolescência dos equipamentos eletroeletrônicos compostos quase que totalmente por circuitos eletrônicos ou alguma parte eletroeletrônica.

A área de informática não era vista tradicionalmente como uma indústria poluidora, até que o acelerado avanço tecnológico aliado ao consumismo das empresas e pessoas de comprar novos aparelhos eletrônicos, mesmo quando os atuais não têm nenhum problema. Com esta crescente demanda e utilização, são descartados erroneamente toneladas de lixo eletrônico no Brasil e no Mundo [4]. E como todo lixo normalmente mal descartado traz consequências negativas, o de equipamentos eletrônicos geram graves problemas ambientais por causa da presença de metais pesados, além da exploração de recursos naturais utilizados na manufatura.

No descarte, ao serem jogados no lixo comum, as substâncias químicas presentes nos eletrônicos, como mercúrio, cádmio, arsênio, cobre, chumbo e alumínio, penetram no solo e nos lençóis freáticos, contaminando plantas e animais por meio da água, e consequentemente os seres humanos pela ingestão desses alimentos, com consequências que vão desde simples dor de cabeça e vomito, até complicações mais sérias, como 


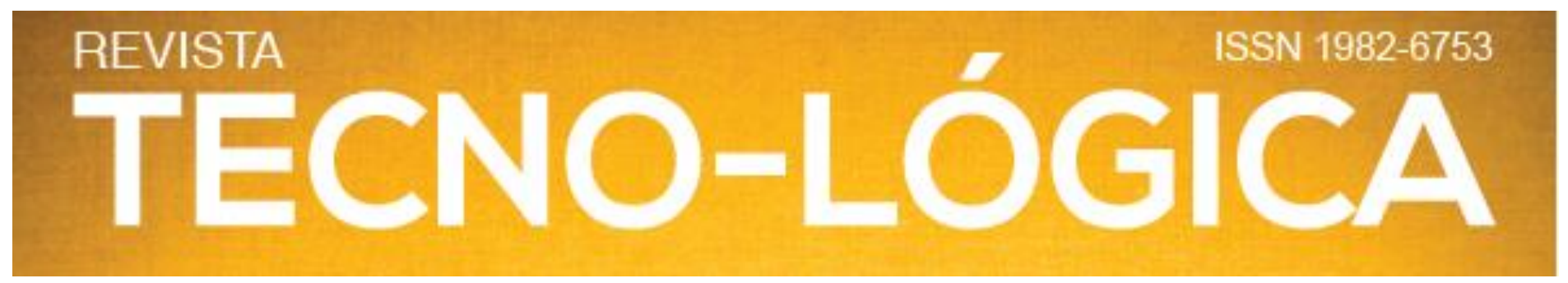

comprometimento do sistema nervoso e surgimento de cânceres por alterações no DNA [8-11]. Na classificação da NBR 10004, indica que são perigosos os resíduos de estanho e os constituintes como cádmio e chumbo, ambos na classe I da resolução do CONAMA (Conselho Nacional do Meio Ambiente) [12-13].

No âmbito Brasil, as pesquisas sobre este espectro da TI Verde são mais voltadas a economia de materiais e energia elétrica, como a otimização através de tecnologias de virtualização. Muitas das ações são paliativas, como por exemplo a compra de créditos de carbono como técnica ao atendimento de leis, tendo-se mais a busca do descarte adequado do lixo eletrônico e não a reciclagem [14-18].

No âmbito internacional, há o uso das práticas e pesquisas do mesmo foco do Brasil [3,5,19,20]. Mas há um esforço maior na otimização dos resultados já obtidos, ao desenvolvimento de métodos e tecnologias para remediação de danos do lixo eletrônico e a reciclagem, tanto em atendimento a uma consciência socioambiental quanto a consciência econômica [6, 21-22]

\section{Normativas, Leis e Empreendedorismo no Brasil}

Para adequar processos e restrições, há as normativas e leis. A normativa principal quando o assunto é ambiental é a ISO 14001, que especifica a Gestão Ambiental dentro de uma corporação. O intuito é desenvolver e praticar regras sustentáveis dentro do ambiente empresarial, e externamente em relação ao que faz como atividade e dos seus recursos eletrônicos, sem agredir ou impactar de forma negativa, ou o mínimo possível, o meio ambiente ao qual a empresa está inserida [23-24].

A lei brasileira que rege sobre o "lixo eletrônico" é a 12.305 de 2 de agosto de 2010 sobre Resíduos Sólidos [25]. Essa legisla genericamente sobre o lixo eletrônico pois sobre este define apenas a "Responsabilidade Compartilhada", onde informa no Artigo 33 que "são obrigados a estruturar e implementar sistemas de logística reversa" para resíduos sólidos "os fabricantes, importadores, distribuidores e comerciantes" e "por todo o ciclo de vida do produto". Este ciclo de vida define-se da compra ao descarte, ou como informa a lei, "mediante retorno dos produtos após o uso pelo consumidor", contemplando a área no inciso VI de "produtos eletroeletrônicos e seus componentes". Ao setor de "Eletroeletrônicos e seus componentes", o Comitê Orientador para a Implantação de Sistemas de Logística Reversa, criado pelo decreto 7.404/10 [26], oficializou em 2019 o acordo onde promete que " $100 \%$ dos produtos coletados deverão ser enviados para a destinação final ambientalmente adequada". A Logística Reversa é citada também em lei mais antiga, a Lei 13.576/09 do Estado de São Paulo. Ambas leis, paulista e a brasileira, fazem com que o fabricante tenha a responsabilidade sobre o produto, podendo ele apenas receber a coleta, ou se quiser, otimizar o descarte e a reciclagem deste produto que produziu.

A Logística Reversa é hoje o principal meio de TI Verde quanto ao descarte [27], que teve seu decreto sobre a obrigatoriedade em relação a produtos eletroeletrônicos de uso doméstico e seus componentes regulamentado em 2020 [28], 10 anos após a instituição da Política Nacional de Resíduos Sólidos [25]. O envolvimento de entidades como ABINEE (Associação Brasileira da Indústria Elétrica e Eletrônica), ABRADISTI (Associação Brasileira da Distribuição de Produtos e Serviços de Tecnologia da Informação), ASSESPRO NACIONAL (Federação das Associações das Empresas Brasileiras de Tecnologia da Informação) e GREEN ELETRON (Gestora para Resíduos de Equipamentos Eletroeletrônicos Nacional), consideram não apenas a potencialidade que o descarte adequado dos produtos apresenta, uma vez que as cidades ficarão menos poluídas, e assim menos tóxicas [29], mas também que o ciclo do produto com "destinação final ambientalmente adequada", potencialize a reciclagem e reinsira materiais na cadeia produtiva [26].

As homologações sobre Resíduos Sólidos e Logística Reversa [25-26] resultaram em empreendimentos já regulamentados com CNAE (Classificação Nacional de Atividades Econômicas), classificando as empresas como de "Coleta, Tratamento e Disposição de Resíduos; Recuperação de Materiais" [30]. Neste setor aumentou em 70\% a quantidade de empresas nos últimos 4 anos, hoje em torno de 140 mil formalizadas, e pouco menos de 1000 com declaração de "eletrônicos" junto a sua descrição em atividades principais de "Coleta de resíduos não-perigosos" [31], ou "Tratamento e disposição de resíduos perigosos" [32] ou "Recuperação de materiais" [33].

\section{Revalorização, Remediação e Reciclagem}

A revalorização age no intuito de proporcionar mais utilização sobre os eletroeletrônicos em desuso, mesmo que saindo da funcionalidade inicial, mas que por outras formas agregue valor e postergue este se tornar "lixo eletrônico". Alguns projetos no Brasil reusam componentes como baterias, leds e displays em trabalhos ligados a robótica, e ao próprio lixo eletrônico, com a revalorização com metareciclagem [34]. Assim os materiais ainda possuem alguma forma de valor dentro do contexto de reuso [35].

O correto descarte e armazenamento evita remediações de danos. Mas quando necessário, o tratamento de contaminação do meio ambiente por metais, que compõem todos os aparelhos eletroeletrônicos tem algumas técnicas. As mais publicadas: a eletrocinética, que usa a dopagem de corrente contínua em placas no sentido do ânodo ao cátodo; separação granulométrica, que por agitação e injeção de reagentes separa os contaminantes e o 


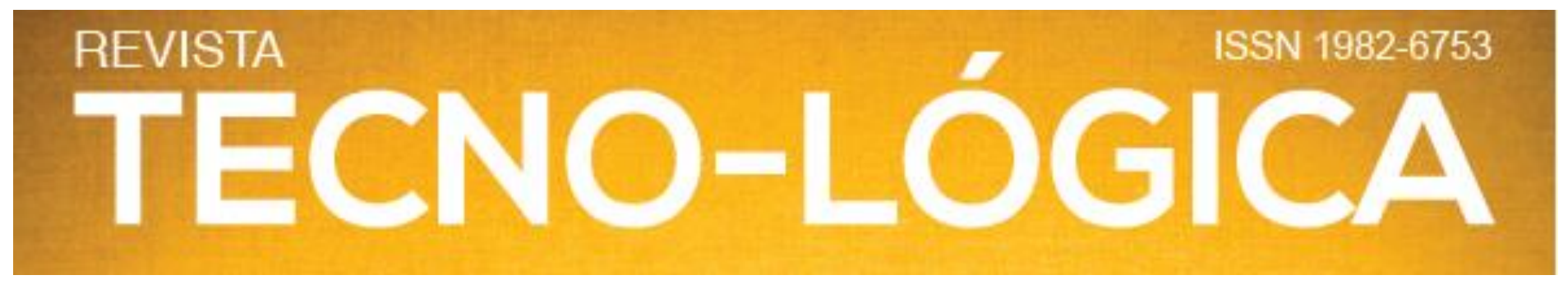

aquecimento controlado, que faz a separação dos metais de acordo as diferentes temperaturas de fusões. Infelizmente todas apresentam alguma perda de eficiência, como de acordo com o tipo de metal e o tempo decorrido, o tipo de sedimentação e a própria volatilização do metal potencializando novas pesquisas [36-38].

A reciclagem é o caminho mais consciente, pois a retirada de matérias-primas, como ouro e paládio é mais efetiva sobre o "lixo eletrônico" do que na mineração [39]. A maioria das metodologias usuais de reciclagem abrangem processos como destruição, triagem, classificação e compactação, excluindo processos mais tecnológicos para a extração de metais que não são muito usuais em larga escala [40]. Na Europa há programas como da Responsabilidade de Produção Estendida que exigem que os fabricantes façam a coleta e reciclagem, mas muito ainda é exportado, legalmente ou ilegalmente, para países menos desenvolvidos [6].

\section{Descarte em Santa Cruz do Sul}

Para a obtenção de dados para análise e discussão, foram realizados contatos telefônicos com 10 empresas com geração ou gestão de lixo eletrônico na área de informática, na cidade de Santa Cruz do Sul, Rio Grande do Sul, Brasil. A metodologia de coleta foi o roteiro de identificação de quem ligava com nome, sendo aluno de doutorado em Tecnologia Ambiental da UNISC (Universidade de Santa Cruz do Sul) e a pergunta principal foi: "O que é feito com o lixo eletrônico que geram/recebem?”. Somaramse ainda informações, quando existentes, sobre quem era o transportador deste resíduo eletrônico, sobre a conscientização dos colaboradores do setor e da população em geral sobre o assunto, em especial este último para o contato com o órgão público.

Quatro empresas de informática da cidade repassaram informações quanto ao descarte de lixo eletrônico. Resumidamente estas empresas, por serem micro ou pequenas empresas e por conseguirem reaproveitar algumas peças, acabam gerando pouco resíduo eletrônico, um número aproximado e informal aproximado de 200 unidades anuais. Elas também incluem uma prática de que os componentes estragados, e substituídos por novos em seus serviços de assistência técnica, são devolvidos aos clientes, para que estes então descartem. Uma das empresas, com filiais em outras cidades, tem uma quantidade maior de lixo eletrônico que as outras e por isso tem um convênio com uma empresa terceirizada que faz o recolhimento e a destinação adequada, conforme mencionado. Outra destas empresas alega que, por causa da baixa quantidade de resíduos, entrega os mesmos na CEPREL.

A CEPREL é a Central de Recebimento de Pneus e Resíduos Eletrônicos, local criado e gerido a cerca de 8 anos pela
SMMASS - Secretaria Municipal de Meio Ambiente, Saneamento e Sustentabilidade. Lá são recebidos e acondicionados todos os resíduos eletrônicos recolhidos na cidade, em pontos de coleta eventuais criados em campanhas, e de forma permanente na própria sede e nas subprefeituras [41-42]. Este local é destinado a ao estoque e destino ambientalmente correto por empresas terceirizadas, onde apesar de estar direcionado ao recolhimento de resíduos apenas de pessoas físicas, muitas pessoas jurídicas deixam o material lá, não atendendo corretamente a Lei de Logística Reversa, 12.305/2010.

O último registro encontrado de forma oficial na mídia é de 2015, onde a CEPREL recebia em média 3 mil quilos de resíduos eletrônicos [41], número compatível atualmente por dados informais colhidos junto a colaboradores da mesma, pois a coleta e envio para destino final fica em torno de 1 caminhão por ano. Esta coleta e destino final é feita por uma empresa terceirizada que atendeu as exigências da Prefeitura Municipal, e que venceu a licitação por menor preço. Aos resultados de buscas na internet e nos registros da prefeitura, não foi encontrado menção ao recolhimento de "resíduo eletrônico", mas a empresa terceirizada que faz isso tem a prerrogativa de "recolhimento de lâmpadas fluorescentes queimadas". O processo de Logística Reversa aplicado no município de Santa Cruz do Sul tem como base a Lei Municipal $n^{\circ} 5.389$, de 3 de abril de 2008. Outro órgão contatado foi a COOMCAT (Cooperativa de Catadores e Recicladores de Santa Cruz do Sul). Esta cooperativa recolhe lixo comumente reciclável, como garrafas pet, latas e papel. Quando eventualmente encontram algo de resíduo eletrônico misturado ao tipo de material que recolhem, levam para a CEPREL.

\section{Revalorização de computadores em Santa Cruz do Sul}

Um dos itens do "lixo eletrônico" são computadores estragados ou em desuso. As pessoas físicas no município os podem levar diretamente na CREPEL, ou em pontos de coletas [41-42]. As jurídicas precisam realizar o correto descarte em particular.

Nas empresas estes computadores são normalmente funcionais e substituídos, principalmente em multinacionais, por causa de políticas de atualização de computadores, as vezes em um prazo médio de 3 anos. Este é o tempo sugerido em programas de ciclo de vida de produtos como da Dell [43], como uma forma estratégica e precoce para evitar problemas ou para melhoria de desempenho.

Com foco nestes computadores e na revalorização, em 2019 foi fundado o BTEC (Banco Social de Tecnologia do Vale do Rio Pardo) [44-45]. Este banco social atua através de voluntários para receber computadores em desuso das empresas, os quais são consertados, incluindo procedimentos como a 


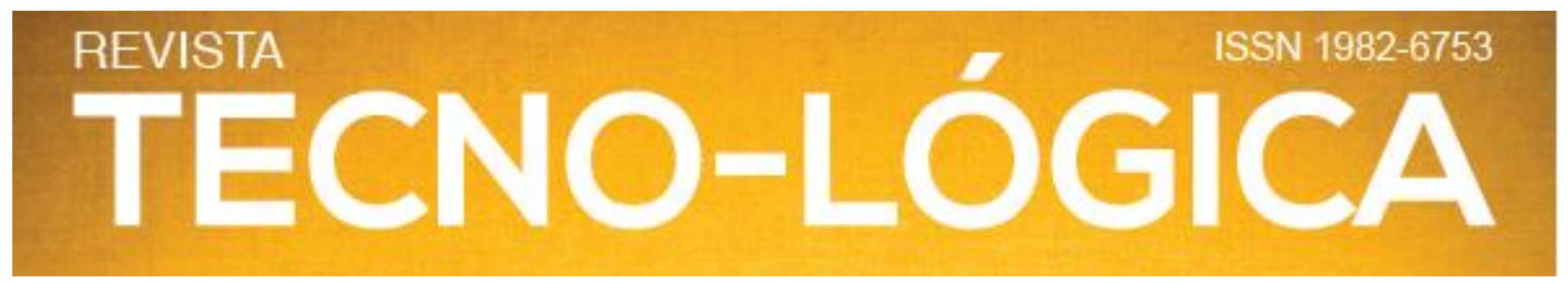

instalação de Softwares Livres para que se tornem novamente funcionais, e após, são doados para escolas e entidades assistenciais da região. Os trabalhos de coleta e revalorização destes computadores começaram no final do ano da sua fundação e teriam as primeiras entregas em 2020, mas por causa da pandemia, suas atividades estão suspensas assim como as escolas que receberiam as primeiras unidades [46].

\section{Considerações Finais}

Observou-se que no Brasil há poucas leis e uma baixa conscientização da população sobre o descarte adequado dos resíduos eletrônicos. Da mesma forma, nas empresas e seus colaboradores, necessitando de atitudes proativas para a conservação do meio ambiente, criando-se uma cultura socioambiental, com maior disseminação e encorajamento de metodologias de TI Verde.

Sobre os contaminantes do resíduo eletrônico na Cidade de Santa Cruz do Sul, observou-se que sem técnicas adequadas perdem-se: a revalorização que poderia ser com o reuso de componentes; a remediação que poderia iniciar pelo correto armazenamento até a descontaminação do meio ambiente; a reciclagem que viria obtendo-se matérias-primas por técnicas como fornos de aquecimento. Assim é necessário estudar e desenvolver novos métodos focados na revalorização, remediação e reciclagem de lixo eletrônico.

Sobre a pesquisa do descarte, notou-se que algumas empresas utilizam a expressão "consciência ambiental" e "TI Verde" em ações de marketing, mas sem ações concretas e recorrentes em prol do meio ambiente. Todavia ações de entidades assistenciais, como o BTEC, pode ser um caminho ambientalmente e socialmente correto, pois as empresas podem descartar de forma adequada computadores em desuso, e estes poderão ser consertados e entregue em escolas para o benefício dos estudantes.

\section{Agradecimentos}

Ao Sr. Valdemar Morais da Rosa da CREPEL e a Sra. Ediane Paulli da CS Informática, por suas prestativas colaborações para a pesquisa, e as empresas e nomes mencionados que autorizaram a sua divulgação. Agradecimentos à ASSEMP (Associação das Empresas de Santa Cruz do Sul) e COMCITI (Conselho Municipal de Ciência e Tecnologia) por apoiar o BTEC.

\section{REVIEW ON ELECTRONIC WASTE AND ITS DESTINATION IN THE CITY OF SANTA CRUZ DO SUL, RS, BRAZIL}

ABSTRACT: Electronic products discarded incorrectly can cause a lot of environmental damage and, consequently, to human health. In this context, a theoretical review was made on the Brazilian laws and standards regarding electronic solid waste, companies operating in the area and the electronic waste disposal assessment in the city of Santa Cruz do Sul, RS, Brazil, in addition to the potential revaluation of locally discarded computers. We verified that there are solutions for electronic waste disposal from individuals and legal entities, especially computer companies in the city, highlighting that for the former there is an appropriate place in the city named CEPREL (Tire and Electronic Waste Collection Center), maintained by the City Hall. Companies have their own processes. We consider as a social benefit that one of the best municipal solution is BTEC (Social Bank of Technology of the Vale do Rio Pardo), which collects disused computers from companies, repairs them and donates to schools and charity entities.

Keywords: Environmental Technology; Electronic Waste; Disposal; Revaluation; Green IT

\section{REFERÊNCIAS}

[1] Molla A, Cooper V, Corbitt B, Deng H, Peszynski K, Yen S. Association for Information Systems AIS Electronic Library (AISeL) E-Readiness to G-Readiness: Developing a Green Information Technology Readiness Framework Recommended Citation. 2008;35. Disponível em: http://aisel.aisnet.org/acis2008/35 Acesso em 15/07/2020.

[2] Watson RT, Boudreau MC, Chen AJ. Information systems and environmentally sustainable development: Energy informatics and new directions for the IS community. MIS Quarterly: Management Information Systems. 2010.

[3] Unhelkar B. Enterprise Green IT Strategy. In: Harnessing Green It: Principles and Practices. 2012.

[4] STEP. Solving the E-Waste Problem (Step) White Paper. One Global Definition of E-waste. Step [Internet]. 2014;3576(June):13. Disponível em: https://www.step-

initiative.org/files/step/_documents/StEP_WP_OneGlobalDefinitionofEwaste_20140603_amended.pdf Acesso em 15/07/2020.

[5] Uddin M, Hindu RC, Alsaqour R, Shah A, Abubakar A, Saba T. Knowledge management framework using green IT to implement sustainable entrepreneur ecosystem. Appl Math Inf Sci. 2015;

[6] Mihai F-C, Gnoni M-G. E-waste Management as a Global Challenge (Introductory Chapter). In: E-Waste in Transition - From Pollution to Resource [Internet]. InTech; 2016. p. 12-9. Disponível em: http://www.intechopen.com/books/e-waste-in-transition-from-pollution-toresource/e-waste-management-as-a-global-challenge-introductory-chapterAcesso em 15/07/2020. 


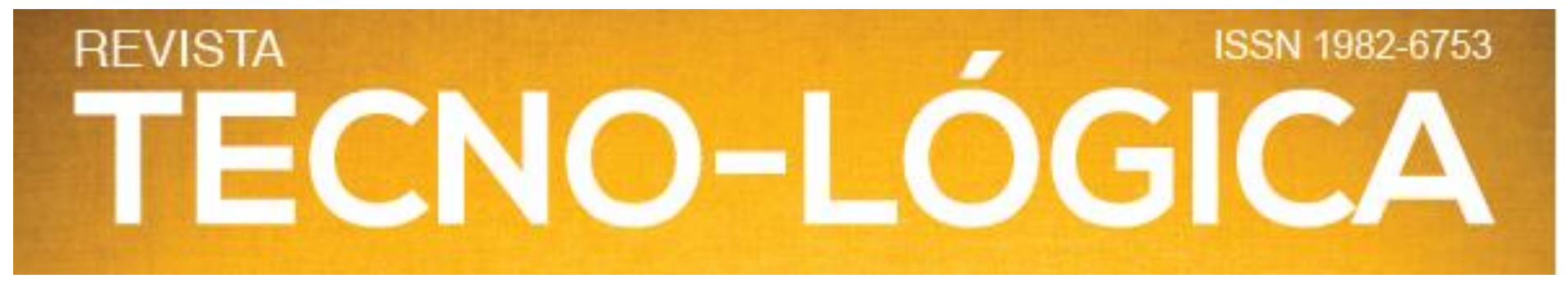

[7] Moraes S de B, Langhi C, Teixeira EP. Tecnologia Da Informação Sustentável (Green It) - O Que É Relevante Para As Empresas Brasileiras? Rev Eletrônica Tecnol e Cult. 2016;18:48-57.

[8] Carpanez J. Dez mandamentos reduzem lixo eletrônico. G1 Tecnol [Internet]. 2007;1-2. Disponível em: https://g1.globo.com/Noticias/Tecnologia/0,,MRP87082-6174,00.html Acesso em $15 / 07 / 2020$

[9] Alabi OA, Bakare AA, Xu X, Li B, Zhang Y, Huo X. Comparative evaluation of environmental contamination and DNA damage induced by electronic-waste in Nigeria and China. Sci Total Environ [Internet]. 2012;423:6272. Disponível em: http://dx.doi.org/10.1016/j.scitotenv.2012.01.056

[10] Liu Q, Cao J, Li KQ, Miao XH, Li G, Fan FY, et al. Chromosomal aberrations and DNA damage in human populations exposed to the processing of electronics waste. Environ Sci Pollut Res. 2009;16(3):329-38.

[11] Grant K, Goldizen FC, Sly PD, Brune MN, Neira M, van den Berg M, et al. Health consequences of exposure to e-waste: A systematic review. Lancet Glob Heal [Internet]. 2013;1(6):e350-61. Disponível em: http://dx.doi.org/10.1016/S2214-109X(13)70101-3 Acesso em 15/07/2020.

[12] Conselho Nacional do Meio Ambiente (CONAMA). Resolução no 313, de 29 de outubro de 2002. Ministério do Meio Ambient. 2002;

[13] ABNT. NBR 10004 - Norma Brasileira sobre Resíduos Sólidos Classificação. Assoc Bras Normas Técnicas. 2004;

[14] Conejero MA, Neves MF. Gestão de créditos de carbono: um estudo multicasos. Rev Adm. 2007;42(2):113-27.

[15] Siqueira ML de, Machado CMC. Atividade extensionista e TI Verde conectando instituição e comunidade. Rev Bras Comput Apl. 2013;5(2):18-27.

[16] Lunardi GL, Alves APF, Salles AC. Desenvolvimento de uma escala para avaliar o grau de utilização da tecnologia da informação verde pelas organizações. Rev Adm. 2014;49(3):591-605.

[17] Pontes F, Giordano F. Práticas De TI Verde em uma Empresa Educacional para Fomentar a Responsabilidade Socioambiental. Rev Gestão Ambient e Sustentabilidade. 2015;4(2):118-26.

[18] Tavarez AS, Dos Santos ECLO, Albertini GD, Lins RB. O Modelo Das 65 Práticas De Ti Verde: a Aceitabilidade Em Um Ambiente Universitário Brasileiro. Universidade Municipal De São Caetano Do Sul; 2016.

[19] Balde CP, Forti V, Gray V, Kuehr R, Stegmann P. The global e-waste monitor 2017 [Internet]. United Nations University. 2017. 1-116 p. Disponível em: http://collections.unu.edu/eserv/UNU:6341/Global-E-

waste_Monitor_2017_electronic_single_pages_.pdf Acesso em 15/07/2020.

[20] Hba R, Bakkas A, El Manouar A, Janati Idrissi MA. Eco-Strategy: Towards a New Generation Managerial Model Based on Green IT and CSR. Int J Comput Sci Inf Technol. 2016;8(2):51-62.

[21] Aung WW. Preserving green computer labs in developing countries with thin technology. In: 2015 IEEE 3rd International Conference on MOOCs, Innovation and Technology in Education (MITE) [Internet]. IEEE; 2015. p. 21-5. Disponível em: http://ieeexplore.ieee.org/document/7375280/ Acesso em $15 / 07 / 2020$

[22] Rajput A, Kumar Raghuwanshi R, Thakur MV. Innovative Green Ict for Environmental Sustainability. Int J Res Comput Appl Robot www.ijrcar.com. 2015;35(5):115-20.

[23] Savita KS, Dominic PDD, Ratnam KA. The role of green IT and IT for green within green supply Chain management: A preliminary finding from ISO14001 companies in Malaysia. In: Lecture Notes in Electrical Engineering. 2015.
[24] Daddi T, Frey M, De Giacomo MR, Testa F, Iraldo F. Macroeconomic and development indexes and ISO14001 certificates: A cross national analysis. J Clean Prod. 2015;

[25] BRASIL. Lei No 12.305 de 2 de agosto de 2010, institui a Política Nacional de Resíduos Sólidos [Internet]. Brasil; 2010 p. 1-19. Disponível em: http://www.planalto.gov.br/ccivil_03/_ato2007-2010/2010/lei/112305.htm Acesso em 15/07/2020

[26] BRASIL. Ministério do Meio Ambiente celebra Acordo Setorial de Eletroeletrônicos. 2019.2 Disponível em: https://www.mma.gov.br/informma/item/15652-minist\%C3\%A9rio-do-meioambiente-celebra-acordo-setorial-de-eletroeletr\%C3\%B4nicos.html Acesso em $15 / 07 / 2020$.

[27] Alves DS, Farina MC. Disposal and reuse of the information technology waste: a case study in a Brazilian university. Eur Bus Rev [Internet]. 2018;30(6):720-34. Disponível em: https://www.emerald.com/insight/content/doi/10.1108/EBR-08-20160117/full/html Acesso em 15/07/2020.

[28] BRASIL. Decreto 10.240 de 12 de fevereiro de 2020, regulamenta a implementação de sistema de logística reversa de produtos eletroeletrônicos e seus componentes de uso doméstico. [Internet]. Inciso VI do caput do art. 33 e o art. 56 Brasil; 2020 p. 1-29. Disponível em: http://www.planalto.gov.br/ccivil_03/_ato2019-2022/2020/decreto/D10240.htm Acesso em 15/07/2020.

[29] Kennedy CA, Ibrahim N, Hoornweg D. Low-carbon infrastructure strategies for cities. Nat Clim Chang [Internet]. 16 de maio de 2014;4(5):343-6. Disponível em: http://www.nature.com/articles/nclimate2160 Acesso em 15/07/2020.

[30] IBGE. Concla - Comissão Nacional de Classificação, Busca online [Internet]. IBGE-Instituo Brasileiro de Geografia e Estatística. 2020. p. 2020. Disponível em: https://concla.ibge.gov.br/busca-onlinecnae.html view $=$ classe $\&$ tipo $=$ cnae $\&$ versao $=10 \&$ classe $=42928$ Acesso em 15/07/2020.

[31] EmpresasCNPJ. Resultados para "Coleta de resíduos não-perigosos" [Internet]. 2020. p. 2-5. Disponível em: https://www.empresascnpj.com/ Acesso em 15/07/2020.

[32] EmpresasCNPJ. Resultados para "Tratamento e disposição de resíduos perigosos" [Internet]. 2020. p. 2-5. Disponível em: https://www.empresascnpj.com/ Acesso em 15/07/2020.

[33] EmpresasCNPJ. Resultados para "Recuperação De Materiais" [Internet]. 2020. p. 2-5. Disponível em: https://www.empresascnpj.com/ Acesso em 15/07/2020.

[34] CMID CM de I de D. Reaproveitamento - CMID [Internet]. 2015. Disponível em: https://cmidsm.wordpress.com/2015/10/25/reaproveitamento/ Acesso em 15/07/2020

[35] Huge Brodin M, Anderson H. Recycling calls for revaluation. Supply Chain Manag An Int J [Internet]. 25 de janeiro de 2008;13(1):9-15. Disponível em: https://www.emerald.com/insight/content/doi/10.1108/13598540810850274/full/h tml Acesso em 15/07/2020.

[36] Clifton J, Mcdonald P, Plater A, Oldfield F. An investigation into the efficiency of particle size separation using Stokes' Law. Earth Surf Process Landforms. 1999;24(8):725-30.

[37] Peng G, Tian G. Using electrode electrolytes to enhance electrokinetic removal of heavy metals from electroplating sludge. Chem Eng J [Internet]. 2010;165(2):388-94. Disponível em: http://dx.doi.org/10.1016/j.cej.2010.10.006

[38] Ho TC, Chu HW, Hopper JR. Metal volatilization and separation during incineration. Waste Manag. 1993;13(5-7):455-66. 


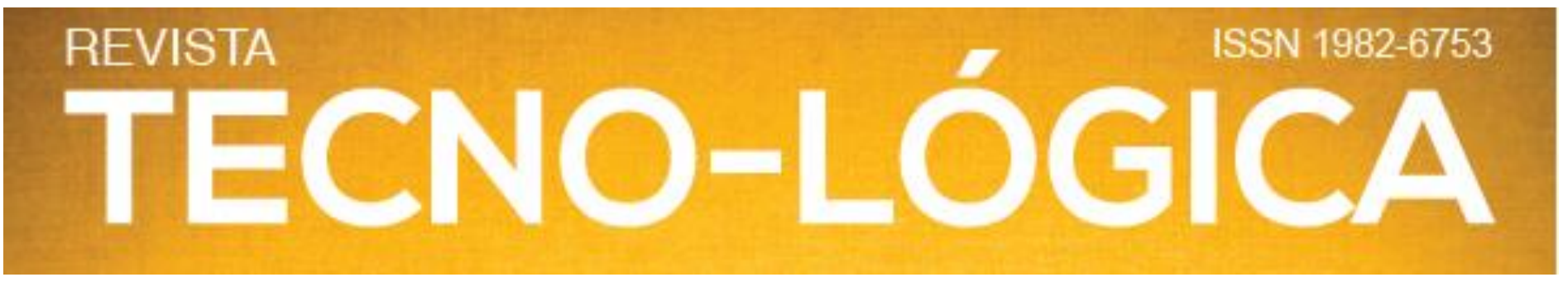

[39] Mmereki D, Li B, Baldwin A, Hong L. The Generation, Composition, Collection, Treatment and Disposal System, and Impact of E-Waste. In: E-Waste in Transition - From Pollution to Resource [Internet]. InTech; 2016. p. 76-104. Disponível em: http://www.intechopen.com/books/e-waste-in-transition-frompollution-to-resource/the-generation-composition-collection-treatment-anddisposal-system-and-impact-of-e-waste Acesso em 15/07/2020.

[40] Vidyadhar A. A Review of Technology of Metal Recovery from Electronic Waste. In: E-Waste in Transition - From Pollution to Resource [Internet]. InTech; 2016. p. 132-69. Disponível em: http://www.intechopen.com/books/e-waste-in-transition-from-pollution-toresource/a-review-of-technology-of-metal-recovery-from-electronic-waste Acesso em 15/07/2020.

[41] Santa Cruz do Sul PM. Secretaria de Meio Ambiente mapeia 16 pontos de descarte irregular [Internet]. 2015. p. 15-7. Disponível em: https://www.santacruz.rs.gov.br/noticias/806/secretaria-de-meio-ambientemapeia-16-pontos-de-descarte-irregular Acesso em 15/07/2020.

[42] Santa Cruz do Sul PM. Subprefeituras recebem lixo eletrônico a partir ... Subprefeituras recebem lixo eletrônico a partir de abril Secretaria de Comunicação Endereço. 2019;14-5. Disponível em: https://www.santacruz.rs.gov.br/noticias/3690/subprefeituras-recebem-lixoeletronico-a-partir-de-abril Acesso em 15/07/2020.

[43] DELL. Serviços para o ciclo de vida dos PCs da Dell. 2020. Disponível em: https://www.delltechnologies.com/pt-pt/pc-lifecycle/index.htm. Acesso em 15/07/2020.

[44] BTEC. BTec - Banco Social de Tecnologia do Vale do Rio Pardo [Internet]. website. 2020 [citado 13 de julho de 2020]. p. 2-4. Disponível em: https://assempscs.com.br/btec/ Acesso em 15/07/2020.

[45] GAZ CG. Banco Social de Tecnologia beneficia escolas públicas e instituições assistenciais [Internet]. 2019. p. 2019-21. Disponível em: http://www.gaz.com.br/conteudos/regional/2019/04/23/144359-

banco_social_de_tecnologia_beneficia_escolas_publicas_e_instituicoes_assistenc iais.html.php Acesso em 15/07/2020.

[46] GAZ CG. Aulas na rede municipal de Santa Cruz estão suspensas a partir de quinta-feira. 2020;50-2. Disponível em: http://www.gaz.com.br/conteudos/regional/2020/03/16/163154-

aulas_na_rede_municipal_de_santa_cruz_estao_suspensas_a_partir_de_quinta_fe ira.html.php Acesso em 15/07/2020. 